\title{
Hypoxia and the heart of poikilotherms
}

\author{
Bohuslav Ostadal MD
}

\author{
B Ostadal. Hypoxia and the heart of poikilotherms. Curr Res \\ Cardiol 2014;1(1):28-32.
}

Hypoxic states originate as a result of an insufficient amount of oxygen supplied to the cardiac cell and the amount actually required by the cell. The dominant factor affecting the supply of oxygen to cardiac structures is governed by the type and overall capacity of the cardiac blood supply. Whereas the heart of adult homeotherms consists entirely of compact musculature with coronary blood supply, the cardiac musculature of poikilothermic animals consists either entirely of the avascular spongious type, supplied by diffusion from the ventricular cavity, or its spongious musculature is covered by an outer compact layer supplied from coronary arteries. As a whole (ie, without distinction between the compact and spongious layer), the adult poikilothermic heart is significantly more tolerant to

$\mathrm{T}$ he most prevalent and, hence, most widely studied contemporary cardiovascular diseases undoubtedly include hypoxic states, which originate as a result of an insufficient amount of oxygen supplied to the cardiac cell and the amount actually required by the cell. The degree of injury depends, however, not only on the intensity and duration of hypoxic stimulus, but also on the level of cardiac tolerance to oxygen deprivation. This particular parameter changes significantly during ontogenetic and phylogenetic development (1). Whereas significant data regarding cardiac tolerance to hypoxia in warm-blooded animals are available, much less is known about the tolerance to oxygen deprivation in the poikilothermic heart. Although it is exceedingly difficult to agree fully with the view that ontogenetic development is a recapitulation of phylogeny, comparative studies (more correct than 'phylogenetic', because the researcher is comparing not the entire evolutionary range but only some classes [ie, a 'pars pro toto' approach]) have made significant contributions to our understanding of the function of the cardiovascular system $(2,3)$. From the point of view of developmental cardiology, the advantage of comparative studies is that they enable the study of selected developmental periods as stable situations, an undertaking that is exceedingly difficult during rapidly changing ontogeny. Unfortunately, core knowledge of cardiac structure and function in lower vertebrates has only rarely been used to gain insight into cardiac function in avian and mammalian ontogeny. Analysis of cardiac tolerance to hypoxia may be a typical example $(1,4)$.

The present review discusses some aspects of the relationships between oxygen-consuming and oxygen-supplying systems in the heart of poikilotherms, as well as the determinants responsible for cardiac tolerance to oxygen deprivation in the poikilothermic heart.

\section{COMPARATIVE ASPECTS OF THE MYOCARDIAL STRUCTURE/BLOOD SUPPLY RELATIONSHIP}

As mentioned above, the oxygen supply of the heart depends on the balance between oxygen-supplying and oxygen-consuming systems. The heart is apparently not able to increase its oxygen supply by any appreciable extent by increasing oxygen extraction from the blood. Thus, the dominant factor affecting the supply of oxygen to cardiac oxygen deprivation compared with the homeothermic heart, probably because of higher anaerobic capacity and differences in systems responsible for calcium handling. The hearts of chronic hypoxia-acclimated poikilotherms maintain maximum performance longer when faced with severe acute hypoxia and recover better than hearts of normoxic animals following an acute hypoxic insult. Whether the protective mechanisms in poikilothermic and homeothermic animals are the same remains to be clarified in future experiments. Thus, the poikilothermic heart represents a unique model for comparison of the tolerance to oxygen deprivation in two precisely defined, developmentally stable layers of the same heart differing in structure, type of blood supply as well as the capacity for energetic metabolism.

\section{Key Words: Blood supply; Heart; Poikilotherms; Tolerance to hypoxia} blood supply. The first coronary vessels appeared in some fish at least 500 million years ago (2). According to Grant and Regnier (5), a critical role in the development of coronary vessels is played by the pericardial ligaments connecting the heart of lower vertebrates with the rest of the organism. These ligaments are well developed in fish and among amphibians in urodels, whereas in anurans and reptiles, they are comprised of only one or two strands, and they disappear completely in mammals. In species in which these ligaments were formed, two types of blood supply exist: the cephalic - in which the coronary arteries arise from the hypobranchial branches and the venous blood flows back into the sinus venosus; and caudal - the extracoronary arteries penetrate the pericardium from the systemic circulation and the venous blood returns to systemic veins. Briefly, the blood supply to the heart is either hilar (coronary vessels) or extrahilar (extracoronary vessels) (6).

The development of coronary arteries during phylogeny is closely related to the transformation of the musculature from a spongy avascular myocardium to a compact myocardium supplied by coronary vessels. Whereas the heart of adult homeotherms consists entirely of compact musculature with coronary blood supply, the cardiac musculature in poikilothermic animals consists either entirely of the spongious type or its spongious musculature is covered by an outer compact layer $(1,5-8)$. The sponge-like musculature is supplied predominantly by diffusion from the intertrabecular spaces. They belong to the chamber cavity and have a continuous endothelial lining. Nevertheless, in some species of fish and reptiles, capillaries can also be found in some trabecels of the sponge-like musculature. There are no structural differences between the capillaries in compact and spongious musculature, nor between capillaries in the heart of cold-blooded and warm-blooded animals $(9,10)$.

There are four general types of myocardial blood supply in the animal kingdom (Figure 1):

1. Spongious musculature only, supplied from the ventricular cavity;

2. An inner spongious layer, covered by an outer compact musculature with a vascular supply;

Institute of Physiology, Academy of Sciences of the Czech Republic, Prague, Czech Republic

Correspondence: Dr B Ostadal, Institute of Physiology, Academy of Sciences of the Czech Rrepublic, Videnska 1083, 4220 Prague 4, Czech Republic.

E-mail ostada@biomed.cas.cz 
3. As in 2, but capillaries are also present in some trabecels of the spongious layer; or

4. Compact musculature only, which is supplied from coronary vessels. The trabecular nature of the spongy myocardium increases the surface area and reduces the diffusion distance for oxygen transfer from luminal blood. Presumably, the thickness of the spongy trabeculae is a compromise between minimizing the distance for oxygen diffusion and maximizing the cross-sectional area for tension development (11). Quantitative analysis of the terminal blood bed (6) revealed that poikilotherms with a low relative heart weight (eg, fish) have significantly lower lacunar capacity than animals with a higher relative heart weight, such as amphibians and reptiles. Kohmoto et al (12) demonstrated a high degree of direct myocardial perfusion from the ventricular cavity in the endocardial region of alligator hearts, thus decreasing the diffusion distance for oxygen.

The myocardial blood supply of certain lower vertebrates appears to be 'primitive' and 'inefficient'. However, the principles behind the development of this design may inform clinicians who treat heart disease (13). Investigators researching treatment for occluded coronary arteries became interested in the spongy myocardium of fish and reptiles. The information obtained represents a significant step toward achieving transmyocardial blood flow in humans. Surgeons have used lasers to punch holes in the left ventricle to permit some oxygenation directly from the luminal blood, a technique known as transmyocardial revascularization (14). This method may improve myocardial perfusion in patients in whom the standard method of revascularization is contraindicated.

\section{DETERMINANTS OF THE DEVELOPMENT OF COMPACT MUSCULATURE WITH VASCULAR SUPPLY} The main determinants of cardiac transformation from the avascular spongy type to the compact type with vascular supply have been questioned. The exclusively spongious type can be observed not only in cyclostomes and some teleost fish but also in some amphibians. On the other hand, the mixed types (compact + spongious) are present in fish $(9,15)$, amphibians $(6)$ and reptiles $(10,16)$. It appears, therefore, that the presence of the compact layer is not related to the phylogenetic position of the poikilothermic heart.

More than 35 years ago, an attempt was made to relate the growth of the compact layer to the physiological factors that determine overall oxygen consumption $(7,9,17)$. These factors are body mass, muscular activity, capacity of oxidative metabolism and maintenance of body temperature. It was found that the myocardium of fish and amphibians with low body weight is spongious, and that the thickness of the compact layer increases with increasing heart and body weight. This relationship was also valid within the same species investigated, carp and turtle (18). The suggestion that the total amount of compact layer is related more to the physiological demand of the species than to its phylogenetic classification was further supported by an extensive survey by Santer and Greer Walker (19). The proportion of the cardiac wall occupied by coronary-supplied compact myocardium varies considerably (eg, in fish between 7\% and 37\% [20,21]), with the highest proportion in very active species (eg, tuna, mackerel, sprat, herring, etc). Moreover, Poupa et al (22) observed an increase in the thickness of the compact myocardium in fish (salmonids) during ontogenetic development. In the turtle, the compact layer composes $55 \%$ of the total cardiac weight (18).

Agnisola and Tota (23), and Tota and Gattuso (24) analyzed the relationship between the ventricular myoarchitecture and hemodynamic conditions in cold-blooded animals. They compared the stroke volume and afterload in different species with various degrees of development of the compact musculature with vascular supply. The entirely spongious frog heart moves relatively large volumes of blood against high pressures. A similar relationship can be observed among different species of fish: from the spongious heart of icefish with a 'volume' pump chamber to that of tuna with mixed (arterial and lacunar) blood supply, as a prototype of a 'pressure' pump. The sedentary

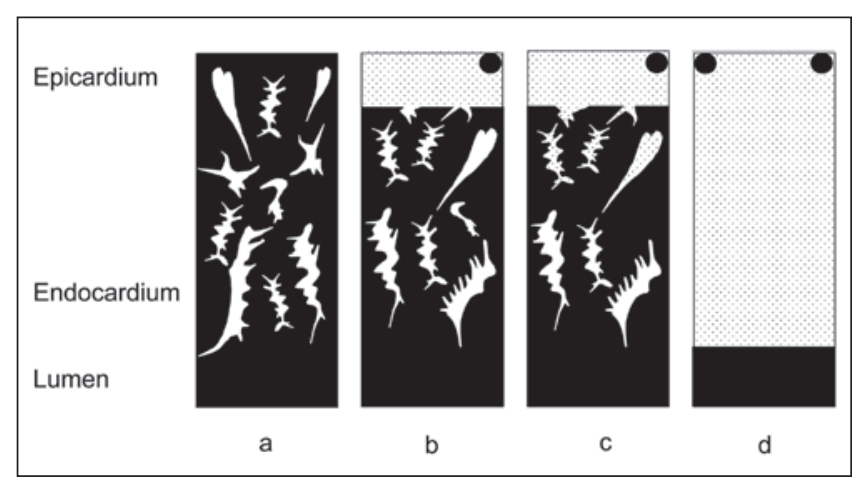

Figure 1) Different types of myocardial blood supply in vertebrates. A Spongious musculature supplied from ventricular lumen. B Inner spongious layer is covered by an outer compact musculature with vascular supply. C As in B, but capillaries are present also in some trabecels of spongious musculature. D Compact musculature supplied from coronary vessels. Adapted from reference 6

ice fish living in polar seas are characterized by the evolutionary loss of hemoglobin and the lack of functionally significant amounts of myoglobin. This fact is compensated for by a high blood volume and low heart rate (approximately 16 beats/min). On the other hand, 'athletic' tuna living in warm tropical water have a cardiovascular system designed to generate a high pressure in high-resistance systemic circulation. The high values are attained, in contrast with ice fish, at a heart rate as high as 120 beats per min. Based on these observations, it may be concluded that generation of higher blood volume requires a higher level of ventricular wall stress, which can be better attained by the development and/or thickening of the compact musculature.

Clark and Rodnick (25) studied the morphometric characteristics of ventricular enlargement during ontogenetic development of rainbow trout. They found that increasing ventricular mass during sexual maturation results from an expansion of both myocardial layers, but growth of the compact layer exceeds that of the spongious musculature. They confirmed the observation of Farell et al (26) that growth of the trout ventricle occurs through a combination of myocyte hypertrophy and hyperplasia.

All of these findings provide additional support for the hypothesis that an increase in the compact layer is necessary for the maintenance of higher blood pressure in larger hearts (application of the law of Laplace to poikilothermic hearts). This hypothesis also means, however, that the primary evolutionary step is not the development of arterial blood supply but the development of the compact layer, necessary for hemodynamic adaptations (ie, for higher blood pressure generation). Vascularization is, thus, the consequence of this evolution because coronary arteries are the only way to supply the compact musculature with blood. The heterogenous heart of cold-blooded animals, thus, offers a unique opportunity to compare the sensitivity of the two well-defined myocardial layers with different pathogenetic conditions including the effect of oxygen deprivation (27).

\section{CARDIAC TOLERANCE TO HYPOXIA IN POIKILOTHERMS}

Hypoxia is a frequently occurring environmental phenomenon in many fresh water and coastal systems, and can be caused by either anthropogenic input or naturally occurring biological and physical factors. The interest in the effect of oxygen deprivation on hearts of poikilotherms has surprisingly increased recently (4). It has been observed that hypoxia in marine waters is not restricted to localized areas, but is more extensive and longer lasting (weeks to months) than previously believed $(28,29)$. These problems become even more serious if large areas are affected by hypoxia for an extended time because fish may not be able to leave these areas (ie, avoidance being the predominant reaction to hypoxia) (30). The understanding of how chronic hypoxia 


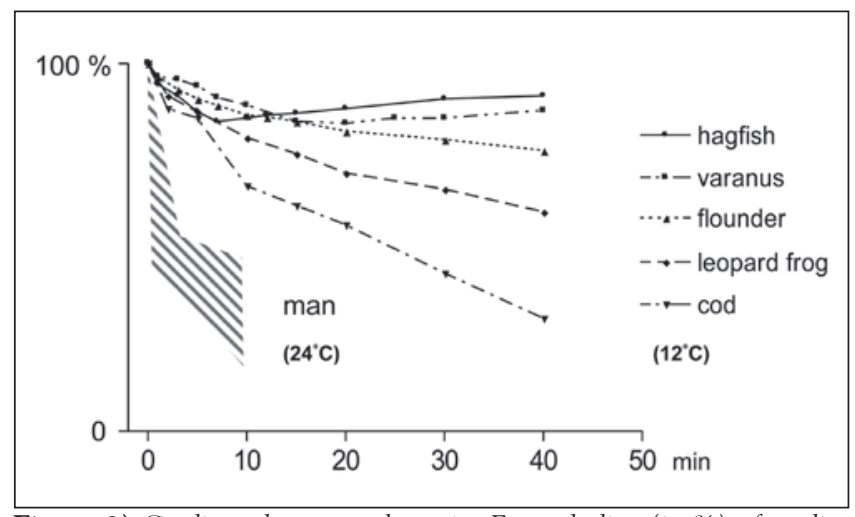

Figure 2) Cardiac tolerance to hypoxia. Force decline (in \%) of cardiac strips in vitro $\left(12^{\circ} \mathrm{C}\right.$ paced 12 min in Ringer with $11.9 \mathrm{mM} \mathrm{HCO}_{3}$ man: $24^{\circ} \mathrm{C}$ paced $\left.4 \mathrm{~min}^{-1}\right)$ when respiratory energy production was stopped by $\mathrm{CN}^{-}(3 \mathrm{mM})$. Adapted from reference 2

affects both swimming performance and cardiovascular function could reveal important information as to whether fish will survive and how well they adapt to hypoxic environments.

Of the experimental models used for studying the effect of oxygen deprivation in homeotherms, only a limited number are suitable for the cold-blooded heart. The absence of, or partially developed coronary circulation (1) excludes, for instance, the possibility of using regional ischemia. The most frequently used models are, therefore, systemic and histotoxic hypoxia. The poikilothermic heart, which is frequently exposed to oxygen deficiency in an aquatic environment, is better equipped biochemically to cope with oxygen deprivation than the mammalian heart (1). Data comparing the sensitivity of the poikilothermic and homeothermic heart to oxygen deficiency are relatively scarce. The anaerobic capacity of cardiac muscle in different chordates from cyclostome (hagfish) to humans has been reviewed by Poupa (2), and Driedzic and Gesser (31) (Figure 2). Force development of isometric cardiac strips in vitro under similar conditions was measured when respiration was blocked by cyanide (histotoxic hypoxia). The highest tolerance to lack of oxygen was observed in the reptilian (monitor lizard) and hagfish heart; the force development was reduced by no more than $30 \%$ and recovered slowly toward initial values. Large differences were found in fish: contractile force in free-swimming cod was reduced by only $50 \%$ (within 45 min after the onset of hypoxia). The highest sensitivity was observed in homeotherms (humans): a decline of contractile force occurred immediately (within $5 \mathrm{~min}$ ) and was irreversible. In poikilotherms, the sensitivity to histotoxic hypoxia was increased on increasing the temperature of the perfusion medium (32). Myocardial cells of poikilotherms, similar to homeotherms, may be irreversibly damaged by hypoxia (33), although highly resistant reptilian heart should be noted as a probable exception (34).

Whereas a large amount of information currently exists on the cardiovascular responses of teleosts to acute hypoxia $(35,36)$, much less is known about the effect of chronic hypoxia on the cardiovascular function of fish. Marques et al (37) found that chronic hypoxia (10\% $\mathrm{O}_{2}$ for three weeks) led to a smaller ventricular outflow tract, reduced lacunae and an increase in the number of cardiac myocyte nuclei per area in the hearts of two teleost species, zebrafish and cichlids. To identify the molecular basis for the adaptation to chronic hypoxia, they profiled the gene expression changes in the heart of adult zebrafish. They have analyzed $>15,000$ different transcripts and found 376 differentially regulated genes, of which 260 showed increased and 116 decreased expression levels. Two notch receptors (notch-2 and notch-3) as well as regulatory genes linked to cell proliferation were upregulated in hypoxic hearts. They observed simultaneous increase in expression of IGF-2 and IGFbp 1 and upregulation of several genes important for protection against reactive oxygen species. Petersen and Gamperl $(38,39)$ studied acclimation of Atlantic cod to chronic hypoxia $\left(8 \mathrm{kPa}\right.$, six to 12 weeks, $\left.10^{\circ} \mathrm{C}\right)$. They observed that acclimation did not affect body and heart weight, or basal in situ cardiac performance under oxygenated conditions. Stroke volume and cardiac output during well-oxygenated conditions were significantly reduced in hypoxia-acclimated animals compared with the normoxic group. Because the same results were obtained in in vivo and in situ experiments, it may be suggested that in vivo cardiac function in hypoxiaacclimated cod was not lower because of alterations in nervous and/or humoral control, but because of the direct effect of chronic hypoxia on the myocardium. The hearts of hypoxia-acclimated animals maintained maximum performance longer when faced with severe acute hypoxia and recovered better than hearts from normoxia-acclimated fish following an acute hypoxic insult. These results suggest that acclimation to chronic hypoxia increases myocardial hypoxic tolerance and are consistent with the substantial body of research that has been conducted on chronically hypoxic mammals (40). There are several mechanisms that have been reported to confer hypoxia tolerance of mammalian hearts adapted to chronic hypoxia. Among these are ATP-sensitive potassium channels, both sarcolemmal and mitochondrial, nitric oxide, HIF- $1 \alpha$ and various protein kinases (41). However, to answer the question of whether the protective mechanisms responsible for increased hypoxic tolerance are the same in poikilothermic and homeothermic animals remains to be clarified in future experiments.

In this connection, the question arises whether the already high tolerance of the poikilothermic heart can be further increased by another protective phenomenon, preconditioning. Overgaard et al (42) and Gamperl et al (43) studied this as yet unanswered question in the highly tolerant hearts of rainbow trout (Oncorhynchus mykiss). They found that hypoxic preconditioning failed to confer any protection against post-hypoxic myocardial dysfunction: inherent myocardial hypoxic tolerance and preconditioning are not additive. These results resemble the situation in highly tolerant neonatal rat hearts (44), their already high hypoxic tolerance was impossible to increase by ischemic preconditioning.

\section{POSSIBLE MECHANISMS}

To understand developmental changes in cardiac tolerance to oxygen deprivation, a basic knowledge of the development of cardiac metabolism is of crucial importance. Decisive differences in cardiac energy metabolism in adult vertebrates are the result of changes in metabolic activities of animal tissues, the most obvious occurring during transition from poikilothermy to homeothermy (31). Thermoregulating mammals and birds have a relative heart weight and total metabolic capacity on average four times higher, and arterial blood pressure six times higher, that that of poikilotherms (2). In terms of anaerobic metabolism, all of the requisite enzymes of glycolysis are routinely detected in vigorous activities. Hexokinase, which catalyzes the first step in the utilization of exogenously supplied glucose, is approxiomately five times more active in the hearts of poikilotherms than homeotherms. The mitochondrial enzymes of the poikilotherm and homeothermic heart are similar in their structure and functional properties, but their activity per mg tissue or tissue protein is considerably lower in cold-blooded hearts (45). The ratio of creatine kinase to cytochrome c oxidase, an approximate estimate of aerobic capacity and cellular energy turnover, is significantly increased in poikilotherms (46). When challenged by hypoxia, this high level may represent an enhanced efficiency to attenuate the impact of depressed energy liberation. Moreover, poikilothermic hearts possesses a high relative glycolytic capacity as indicated by a high pyruvate kinase-tocytochrome c oxidase ratio.

Significant metabolic differences also exist between the compact and the spongious layer of the poikilothermic heart (18). The activities of enzymes that are connected with aerobic oxidation (citrate synthase, malate dehydrogenase) and glucose phosphorylation (hexokinase) are higher in the spongious than in the compact layer; comparable results were obtained by Clark and Rodnick (25). The 
spongy myocardium, more likely to receive high levels of metabolites and highly variable oxygen levels, thus appears to be more equipped for aerobic metabolism than the compact tissue (47). Similarly, the content of phospholipids is higher in the spongious musculature, the greatest difference being in the content of diphosphatidylglycerol (48). Furthermore, Maresca et al (49) and Greco et al (50) have demonstrated that differences in enzyme activities are accompanied by different mitochondrial populations in the two layers. It is interesting to note that myosin ATPase activity is significantly higher in the compact musculature compared with the spongious layer in the carp heart. Because this ATPase activity is known to be related to the speed of contraction, a higher contraction velocity of the compact layer could also be expected (18).

Furthermore, compared with the homeothermic heart, the poikilothermic heart is resistant to the calcium paradox. Because it is well known that calcium plays an important role in the development of cardiac hypoxic injury, the phylogenetic differences in systems involved in calcium handling must be taken into consideration as a possible explanation for the difference in the sensitivity of poikilothermic hearts to oxygen deprivation. The cardiac coupling mechanism in lower vertebrates relies heavily on sarcolemmal calcium fluxes, whereas the sarcoplasmic reticulum is of little or no importance in amphibians (51). In many fish species, the sarcoplasmic reticulum certainly conforms to this amphibian pattern, but there are other species in which the sarcoplasmic reticulum is undoubtedly better developed $(15,52)$. On the other hand, a t-tubular system has never been observed in fish myocardial cells and, thus, the concept of functional couplings between cisternae and $t$-tubules in mammals and birds does not apply to myocardial cells in fish. The functional significance of myocardial sarcoplasmic reticulum in poikilotherms is unclear and may differ between the atrium and ventricle (31).

\section{CONCLUSIONS}

It appears that the adult poikilothermic heart as a whole (ie, without distinction into the compact and spongious layers) is significantly more tolerant to oxygen deprivation than the homeothermic heart, probably because of higher anaerobic capacity and differences in systems responsible for calcium handling. The important question regarding the relationship between the type of myocardial blood supply (lacunar versus coronary) and sensitivity to oxygen deprivation has, unfortunately, not been addressed in experimental studies and remains to be answered.

What can we gain from studying the heart of lower vertebrates for analysis of pathogenetic mechanisms involved in the hypoxic tolerance of the immature heart? Their cardiovascular system, which in many superficial ways resembles anatomically different ontogenetic periods of homeotherms, shows significant morphological and functional diversity. The poikilothermic heart represents a unique model for comparison of the tolerance to oxygen deprivation in two precisely defined, developmentally stable layers (compact, spongious) of the same heart differing in their structure, type of blood supply (lacunar, coronary) as well as the capacity of energetic metabolism.

ACKNOWLEDGEMENT: This study was supported by institutional grant RVO: 67985823.

\section{REFERENCES}

1. Ostadal B, Ostadalova I, Dhalla NS. Development of cardiac sensitivity to oxygen deficiency: Comparative and ontogenetic aspects. Physiol Rev 1999;79:635-59.

2. Poupa O. Heart story: A view to the past. In: Ostadal B, Dhalla NS, eds. Heart Function in Health and Disease. Boston, Dordrecht, London: Kluwer Academic Publishers, 1993:3-22.

3. Burggren WW, Wartburton SJ. Patterns of form and function in developing hearts: Contributions from non-mammalian vertebrates. Cardioscience 1994;5:183-91.

4. Ostadal B. Comparative aspects of cardiac adaptation. In: Ostadal B, Dhalla NS, eds. Cardiac Adaptations. New York, Dordrecht, Heidelberg, London: Springer, 2013:3-18.

5. Grant RT, Regnier M. The comparative anatomy of the cardiac coronary vessels. Heart 1926;13:283.

6. Ostadal B, Rychter Z, Poupa O. Comparative aspects of the development of the terminal vascular bed in the myocardium. Physiol bohemoslov 1970;19:1-7.

7. Poupa O, Ostadal B. Experimental cardiomegalies and "cardiomegalies" - in free-living animals. Ann NY Acad Sci 1969;156:445-68.

8. Ostadal B, Schiebler TH, Rychter Z. Relations between development of the capillary wall and myoarchitecture of the rat heart. In: Cristofalo VJ, Holeckova E, eds. Cell Impairment in Aging and Development. Adv Exp Med Biol 1975;53:375-88.

9. Ostadal B, Schiebler TH. Über die terminale Strombahn in Fischherzen. Z Anat Entwickl-Gesch 1971;34:101-10.

10. Ostadal B, Schiebler TH. Die terminale Strombahn in Herzen der Schildkröte (Testudo Hermanni). Z Anat Entwickl-Gesch 1971;134:111-6.

11. Davie PS, Farell AP. The coronary and luminal circulations of the myocardium of fishes. Can J Zool 1991;69:1993-2001.

12. Kohmoto T, Argenziano M, Yamamoto N, et al. Assesment of transmyocardial perfusion in alligator hearts. Circulation 1997;95:1585-91.

13. Brainerd E. Efficient fish not faint-hearted. Nature 1997;389:229-30.

14. Horvath KA, Mannting F, Cummings N, et al. Transmyocardial laser revascularization: Operative techniques and clinical results at two years. J Thorac Cardiovasc Surg 1996;111:1047-53.

15. Santer RM. Morphology and innervation of the fish heart. Adv Anat Embryol Cell Biol 1985;89:1-102.
16. Brady AJ, Dubkin CH. Coronary circulation in the turtle ventricle. Comp Biochem Physiol 1964;13:119-28.

17. Poupa O, Rakusan K, Ostadal B. The effect of physical activity upon the heart of vertebrates. Med Sport 1970;4:202-33.

18. Bass A, Ostadal B, Pelouch V, et al. Differences in weight parameters, myosin ATPase activity and the enzyme pattern of energy supplying metabolism between the compact and spongious cardiac musculature of carp (Cyprinus carpio) and turtle (Testudo horsfieldi). Pflügers Arch 1973;343:65-77.

19. Santer RM, Greer Walker M. Morphological studies on the ventricle of teleost and elasmobranch hearts. J Zool (Lond) 1980;190:259-72.

20. Santer RM, Greer Walker M, Emerson L, et al. On the morphology of the heart ventricle in marine teleost fish (Teleostei). Comp Biochem Physiol 1983;76:453-9.

21. Sanchez-Quintana D, Hurle JM. Ventricular myocardial architecture in marine fishes. Anat Record 1987;217:263-73.

22. Poupa O, Gesser H, Jonsson S, et al. Coronary-supplied compact shell of ventricular myocardium in salmonids: Growth and enzyme pattern. Comp Biochem Physiol 1974;48:85-95.

23. Agnisola C, Tota B. Structure and function of the fish cardiac ventricle: Flexibility and limitations. Cardioscience 1994;5:145-53.

24. Tota B, Gattuso A. Heart ventricle pump in teleost and elasmobranchs: A morphodynamic approach. J Exp Zoo 1996;275:162-71.

25. Clark RJ, Rodnick KJ. Morphometric and biochemical characteristics of ventricular hypertrophy in male rainbow trout (Oncorhynchus mykiss). J Exp Biol 1998;201:1541-52.

26. Farell AP, Hammons AM, Graham MS, et al. Cardiac growth in rainbow trout Salmo gairdneri. Can J Zool 1988;66:2368-73.

27. Ostadal B, Pelouch V, Bass A, et al. Adaptation of the poikilothermic heart to catecholamine-induced overload. In: Takeda N, Nagano M, Dhalla NS, eds. The Hypertrophied Heart. Boston: Kluwer Academic Publishers, 2000:217-26.

28. Bell GW, Eggleston DB. Species-specific avoidance responses by blue crabs and fish to chronic and episodic hypoxia. Mar Biol 2005; 146:761-70.

29. Gilbert D, Sundby B, Gobeil C, et al. A seventy-two year record of diminishing deep water oxygen in the St Lawrence estuary: The northwest Atlantic connection. Limnol Oceanogr 2005;50:1654-66. 
30. Claireaux G, Webber DM, Kerr SR, et al. Physiology and behaviour of free-swimming atlantic cod (Gadus morhua) facing fluctuating salinity and oxygenation conditions. J Exp Biol 1995;198:61-9.

31. Driedzic WR, Gesser H. Energy metabolism and contractility in ectothermic vertebrate hearts: Hypoxia, acidosis and low temperature. Physiol Rev 1994;74:221-58.

32. Carlsten A, Poupa O, Volkman R. Cardiac lesions in poikilotherms by catecholamines. Comp Biochem Physio 1983;76:567-81.

33. Lennard $R$, Huddart $H$. The effect of hypoxic stress on the fine structure of the flounder heart (Platichthys flesus). Comp Biochem Physiol 1992;101:723-32.

34. Wasser JS, Meinertz EA, Chang SY, et al. Metabolic and cardio dynamic responses of isolated turtle hearts to ischemia and reperfusion. Am J Physiol 1992;262:R437-43.

35. Bushnell PG, Steffensen JF, Johansen K. Oxygen consumption and swimming performance in hypoxia-acclimated rainbow trout, Salmo gairdneri. J Exp Biol 1984;113:225-35.

36. Sandblom E, Axelsson M. Effects of hypoxia on the venous circulation in rainbow trout (Oncorhynchus mykiss). Comp Biochem Physiol 2005;140A:233-9.

37. Marques IJ, Leito JT, Spaink HP, et al. Transcriptome analysis of the response to chronic constant hypoxia in zebrafish hearts. J Comp Physiol B Biochem 2008;178:79-92.

38. Petersen LH, Gamperl AK. Effect of acute and chronic hypoxia on the swimming performance, metabolic capacity and cardiac function of Atlantic cod (Gadus morhua). J Exp Biol 2010a;213:808-19.

39. Petersen LH, Gamperl AK. In situ cardiac function in Atlantic cod (Gadus morhua): Effects of acute and chronic hypoxia. J Exp Biol 2010b;213:820-30.

40. Ostadal B, Kolar F. Cardiac adaptation to chronic high altitude hypoxia. Respir Physiol Neurobiol 2007;158: 224-36.

41. Kolar F, Ostadal B. Molecular mechanisms of cardiac protection by adaptation to chronic hypoxia. Physiol Res 2004;53:S3-13.

42. Overgaard J, Stecyk JA, Gesser H, et al. Preconditioning stimuli do not benefit the myocardium of hypoxia-tolerant rainbow trout (Oncorhynchus mykiss). J Comp Physiol B 2004;174:329-40.
43. Gamperl AK, Faust HA, Dougher B, et al. Hypoxia tolerance and preconditioning are not additive in the trout (Oncorhynchus mykiss) heart. J Exp Biol 2004;207:2497-505.

44. Ostadalova I, Ostadal B, Kolar F, et al. Tolerance to ischemia and ischemic preconditioning in neonatal rat heart. J Mol Cell Cardiol 1998;30:857-65

45. Hulbert AJ, Else PL. Evolution of mammalian endothermic metabolism: Mitochondrial activity and cell composition. Am J Physiol 1989;256:R63-9.

46. Christensen M, Hartmund T, Gesser H. Creatine kinase, energyrich phosphates and energy metabolism in heart muscle of different vertebrates. J Comp Physiol B 1994;164:118-23.

47. Tota B. Vascular and metabolic zonantion in the ventricular myocardium of mammals and fishes. Comp Biochem Physiol 1983;76A:423-38.

48. Drnkova J, Novakova O, Pelouch V, et al. Phospholipid content in the compact and spongious musculature of the carp heart (Cyprinus carpio). Physiol Bohemoslov 1985;34:381-4.

49. Maresca B, Modigh M, Servillo L, et al. Different temperature dependencies of oxidative phosphorylation in the inner and outer layers of tuna heart ventricle. J Comp Physiol Psychol 1976;105:167-72.

50. Greco G, Martino G, Tota B. Further characterization of two mitochondrial populations in tuna heart ventricle. Comp Biochem Physiol 1982;71B:71-5.

51. McLeod AG, Shen ACY, Cambell KP. Frog cardiac calsequestrin. Identification, characterization, and subcellular distribution in two structurally distinct regions of peripheral sarcoplasmic reticulum in frog ventricular myocardium. Circ Res 1991;69:346-59.

52. Moller-Nielsen T, Gesser H. Sarcoplasmic reticulum and excitationcontraction copupling at 20 and $10^{\circ} \mathrm{C}$ in rainbow trout myocardium. J Comp Physiol B Biochem Syst Envirom Physiol 1992;162:526-34. 